\title{
End-of-discharge and End-of-life Prediction in Lithium-ion Batteries with Electrochemistry-based Aging Models
}

\author{
Matthew Daigle* \\ NASA Ames Research Center, Moffett Field, CA, 94035, USA \\ Chetan S. Kulkarni ${ }^{\dagger}$ \\ Stinger Ghaffarian Technologies Inc., NASA Ames Research Center, Moffett Field, CA, 94035, USA
}

\begin{abstract}
As batteries become increasingly prevalent in complex systems such as aircraft and electric cars, monitoring and predicting battery state of charge and state of health becomes critical. In order to accurately predict the remaining battery power to support system operations for informed operational decision-making, age-dependent changes in dynamics must be accounted for. Using an electrochemistry-based model, we investigate how key parameters of the battery change as aging occurs, and develop models to describe aging through these key parameters. Using these models, we demonstrate how we can $(i)$ accurately predict end-of-discharge for aged batteries, and ( $i i)$ predict the end-of-life of a battery as a function of anticipated usage. The approach is validated through an experimental set of randomized discharge profiles.
\end{abstract}

\section{Introduction}

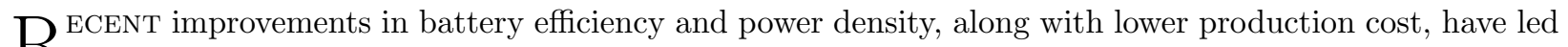
Rto an increased prevalence in many complex systems, such as smartphones, aircraft, and electric cars. In such applications, a critical function is to accurately predict the end-of-discharge (EOD) in order to support operational decision-making. ${ }^{1-3}$ However, EOD is a direct function of battery age; an older battery will have diminished capacity, and, hence, a faster discharge rate. ${ }^{4,5}$ Therefore, it is essential to maintain an accurate model of battery capabilities over several years of usage, for both EOD and end-of-life (EOL) prediction.

While some work has been done in understanding the processes and dynamics underlying battery aging, ${ }^{6-8}$ very little has been done in attempting to estimate battery age, online and in real-time, and using that estimate to obtain condition-based predictions of battery discharge and life. Some approaches, such as Saha et al., ${ }^{4}$ measure the capacity over a sequence of reference discharge cycles (complete discharge at a reference current), and fit some empirical expression to the resulting capacity values. With a reference discharge cycle, capacity is measured simply by determining how long it takes to reach a voltage threshold that defines EOD, and multiplying that by the reference discharge current to obtain a capacity rating in Ah. Capacity measured in this way is dependent on the reference current, because, at a given age, a battery will have a lower Ah rating when the reference current is increased, and higher one when the reference current is decreased, i.e., the relationship between discharge current and discharge time is nonlinear. Further, if the battery is allowed to rest sufficiently in between discharges, the apparent capacity will increase. ${ }^{1}$ Thus, there are two significant problems with such an approach. First, measuring capacity in this way is not an accurate description of battery age, since the Ah rating will depend on the current (and other factors such as temperature), unless such reference conditions can be kept constant (which in an operational environment is not practical). Second, reference discharges are not available in real battery usage; real usage is complex and often unpredictable.

\footnotetext{
${ }^{*}$ Research Computer Scientist, Intelligent Systems Division, Discovery and Systems Health Technical Area.

$\dagger$ Research Engineer III, Intelligent Systems Division, Discovery and Systems Health Technical Area, AIAA Member.
} 
In contrast, our goal is to develop an approach to estimate battery age that can be used in everyday online usage, and does not require reference cycles. Our previous work towards that end used an unscented Kalman filter (UKF) to estimate jointly the battery state and aging parameters online ${ }^{5}$ using an electrochemistry model. ${ }^{1}$ However, the estimation problem in this case is difficult because minor changes in aging parameters over the course of a single cycle can easily be hidden in the data, and corrected simply by updating the state estimates and keeping the parameter estimates static. Essentially, the dynamics of the aging parameters are at a much slower time scale than that of the battery states, so it is difficult to estimate them jointly with the states. Instead, in this work, we separate the estimation task into two phases, a real-time phase in which we estimate only the battery states, assuming aging parameters are known, and a cycle-time phase, in which the aging parameter estimates are updated after every cycle. We assume here that the usage context is that of a battery that undergoes distinct charge and discharge cycles, with variable loads, where aging parameters can be updated based on all the data in a given discharge cycle. This kind of usage context can be found, for example, in electric aircraft, where the aircraft battery is charged pre-flight, and discharges a significant amount during flight before landing. ${ }^{9,10}$

The paper is organized as follows. Section II presents the model-based prognostics framework and the prognostics architecture. Section III reviews the electrochemistry-based modeling for battery discharge. Section IV presents the battery aging models. Section V describes the estimation approaches. Section VI discusses end-of-discharge and end-of-life prediction. Section VII concludes the paper.

\section{Model-based Prognostics}

We formulate the EOD and EOL prediction problems using a model-based prognostics framework. In this section, we formulate the prognostics problem, according to. ${ }^{11}$ We then provide a computational architecture for battery EOD and EOL prognostics.

\section{A. Problem Formulation}

We assume the system model may be generally defined as

$$
\begin{aligned}
\mathbf{x}(k+1) & =\mathbf{f}(k, \mathbf{x}(k), \boldsymbol{\theta}(k), \mathbf{u}(k), \mathbf{v}(k)), \\
\mathbf{y}(k) & =\mathbf{h}(k, \mathbf{x}(k), \boldsymbol{\theta}(k), \mathbf{u}(k), \mathbf{n}(k)),
\end{aligned}
$$

where $k$ is the discrete time variable, $\mathbf{x}(k) \in \mathbb{R}^{n_{x}}$ is the state vector, $\boldsymbol{\theta}(k) \in \mathbb{R}^{n_{\theta}}$ is the unknown parameter vector, $\mathbf{u}(k) \in \mathbb{R}^{n_{u}}$ is the input vector, $\mathbf{v}(k) \in \mathbb{R}^{n_{v}}$ is the process noise vector, $\mathbf{f}$ is the state equation, $\mathbf{y}(k) \in \mathbb{R}^{n_{y}}$ is the output vector, $\mathbf{n}(k) \in \mathbb{R}^{n_{n}}$ is the measurement noise vector, and $\mathbf{h}$ is the output equation. ${ }^{a}$ The unknown parameter vector $\boldsymbol{\theta}(k)$ is used to capture explicit model parameters whose values are unknown and time-varying stochastically.

The goal of prognostics is to predict the occurrence of some event $E$ that is defined with respect to the states, parameters, and inputs of the system. We define the event as the earliest instant that some event threshold $T_{E}: \mathbb{R}^{n_{x}} \times \mathbb{R}^{n_{\theta}} \times \mathbb{R}^{n_{u}} \rightarrow \mathbb{B}$, where $\mathbb{B} \triangleq\{0,1\}$, changes from the value 0 to 1 . That is, the time of the event $k_{E}$ at some time of prediction $k_{P}$ is defined as

$$
k_{E}\left(k_{P}\right) \triangleq \inf \left\{k \in \mathbb{N}: k \geq k_{P} \wedge T_{E}(\mathbf{x}(k), \boldsymbol{\theta}(k), \mathbf{u}(k))=1\right\} .
$$

In this paper, we have two separate prognostics problems, and two corresponding system models, one for EOD and one for EOL. For EOD prediction, the event $E$ represents EOD and is determined by a voltage threshold; the battery is considered to be at EOD when the voltage is less than the low voltage limit. In this case, we will assume there are no unknown parameters (i.e., $\boldsymbol{\theta}=\varnothing$ ). For EOL prediction, the event $E$ represents EOL and is determined by a capacity threshold for a reference discharge; the battery is considered to be at EOL when the capacity is less than the given lower capacity limit.

\section{B. Prognostics Architecture}

In the model-based prognostics architecture, ${ }^{12,13}$ there are two sequential problems, $(i)$ the estimation problem, which determines a joint state-parameter estimate $p\left(\mathbf{x}(k), \boldsymbol{\theta}(k) \mid \mathbf{Y}_{k_{0}}^{k_{P}}\right)$ based on the history of observations up to time $k$, denoted as $\mathbf{Y}_{k_{0}}^{k}$, and (ii) the prediction problem, which determines at prediction time $k_{P}$,

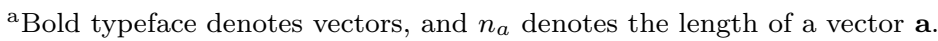




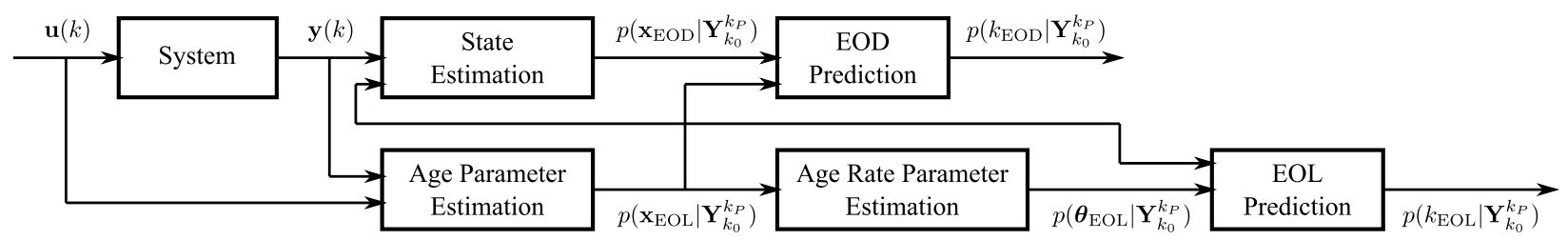

Figure 1. Battery EOD and EOL prognostics architecture.

using $p\left(\mathbf{x}(k), \boldsymbol{\theta}(k) \mid \mathbf{Y}_{k_{0}}^{k_{P}}\right), p\left(\mathbf{U}_{k_{P}}\right), p\left(\mathbf{V}_{k_{P}}\right)$, and $p\left(\boldsymbol{\Theta}_{k_{P}}\right)$, the probability distribution $p\left(k_{E}\left(k_{P}\right) \mid \mathbf{Y}_{k_{0}}^{k_{P}}\right)$. Here, $\mathbf{U}_{k_{P}}$ denotes the future system inputs from $k_{P}$ on, $\mathbf{V}_{k_{P}}$ denotes the future process noise values from $k_{P}$ on, and $\boldsymbol{\Theta}_{k_{P}}$ denotes the future unknown parameter values from $k_{P}$ on.

In this paper, we will assume $\boldsymbol{\Theta}_{k_{P}}$ is known exactly, $\mathbf{V}_{k_{P}}$ is zero, and $\mathbf{U}_{k_{P}}$ is known exactly, in order to focus on validation of the aging models and the performance of the associated predictions. Dropping these assumptions will be addressed in future work. A general framework for dealing with all sources of uncertainty in prognostics is discussed by Sankararaman et al. ${ }^{14}$

The overall combined EOD/EOL prognostics architecture is shown in Fig. 1. In discrete time $k$, the system is provided with inputs $\mathbf{u}(k)$ (current) and provides measured outputs $\mathbf{y}(k)$ (voltage). There are two models used for the two different prediction problems: one for EOD (with subscript EOD), and one for EOL (with subscript EOL). The age parameter estimation block estimates the states for the EOL model, $\mathbf{x}_{\mathrm{EOL}}(k)$, based on the data from the previous discharge cycle. In the context of the EOD model, $\mathbf{x}_{\mathrm{EOL}}(k)$ are the aging parameters and assumed to be constant for a given discharge. The state estimation block estimates states for the EOD model, $\mathbf{x}_{\mathrm{EOD}}(k)$, using the latest estimates of the aging parameters. EOD prediction computes the probability distribution of the EOD time, $k_{\mathrm{EOD}}$, using the latest state estimate and aging parameter estimates. The aging rate parameters, $\boldsymbol{\theta}_{\mathrm{EOL}}(k)$ for the EOL model are estimated based on the estimated aging parameters over past discharge cycles, and parameterize how quickly the aging parameters change in time. EOL prediction computes the probability distribution of the EOL time, $k_{\mathrm{EOD}}$, using the current estimates of the aging and aging rate parameters.

We next describe the EOD and EOL models, followed by descriptions of the estimation and prediction algorithms for these models.

\section{Battery Discharge Modeling}

A battery is a collection of electrochemical cells that convert between chemical and electrical energy. Each cell consists of a positive electrode and a negative electrode with electrolyte. In this paper, we focus on Li-ion cells. The electrolyte enables lithium ions $\left(\mathrm{Li}^{+}\right)$to diffuse between the positive and negative electrodes. The lithium ions insert or deinsert from the active material depending upon the electrode and whether the active process is charging or discharging. ${ }^{15}$

In this work, we use an electrochemistry-based model developed for Li-ion cells in our previous work. ${ }^{1}$ Unlike other electrochemistry-based models that rely on complex partial differential equations that are unsuitable for online estimation and prediction algorithms, this model uses only ordinary differential equations, and is fast enough for real-time use. In this section, we briefly summarize this model and describe its key features.

The voltage terms of the battery are expressed as functions of the amount of charge in the electrodes. Each electrode, positive (subscript $p$ ) and negative (subscript $n$ ), is split into two volumes, a surface layer (subscript $s$ ) and a bulk layer (subscript $b$ ). The differential equations for the battery describe how charge moves through these volumes. The charge $(q)$ variables are described using

$$
\begin{aligned}
& \dot{q}_{s, p}=i_{a p p}+\dot{q}_{b s, p} \\
& \dot{q}_{b, p}=-\dot{q}_{b s, p}+i_{a p p}-i_{a p p} \\
& \dot{q}_{b, n}=-\dot{q}_{b s, n}+i_{a p p}-i_{a p p} \\
& \dot{q}_{s, n}=-i_{a p p}+\dot{q}_{b s, n},
\end{aligned}
$$

where $i_{a p p}$ is the applied electric current The $\dot{q}_{b s, i}$ term describes diffusion from the bulk to surface layer for 
electrode $i$, where $i=n$ or $i=p$ :

$$
\dot{q}_{b s, i}=\frac{1}{D}\left(c_{b, i}-c_{s, i}\right),
$$

where $D$ is the diffusion constant. The $c$ terms are Li-ion concentrations:

$$
\begin{aligned}
c_{b, i} & =\frac{q_{b, i}}{v_{b, i}} \\
c_{s, i} & =\frac{q_{s, i}}{v_{s, i}},
\end{aligned}
$$

Here, $c_{v, i}$ is the concentration of charge in electrode $i$, and $v_{v, i}$ is the total volume of charge storage capability. We define $v_{i}=v_{b, i}+v_{s, i}$. Note now that the following relations hold:

$$
\begin{aligned}
q_{p} & =q_{s, p}+q_{b, p} \\
q_{n} & =q_{s, n}+q_{b, n} \\
q^{\max } & =q_{s, p}+q_{b, p}+q_{s, n}+q_{b, n} .
\end{aligned}
$$

We can also express mole fractions $(x)$ based on the $q$ variables:

$$
\begin{aligned}
x_{i} & =\frac{q_{i}}{q^{\max }}, \\
x_{s, i} & =\frac{q_{s, i}}{q_{s, i}^{\max }}, \\
x_{b, i} & =\frac{q_{b, i}}{q_{b, i}^{\max }},
\end{aligned}
$$

where $q^{\max }=q_{p}+q_{n}$ refers to the total amount of available Li-ions. It follows that $x_{p}+x_{n}=1$. For Li-ion batteries, when fully charged, $x_{p}=0.4$ and $x_{n}=0.6$. When fully discharged, $x_{p}=1$ and $x_{n}=0 .{ }^{16}$

The overall battery voltage $V(t)$ consists of several electrochemical potentials. At the positive current collector is the equilibrium potential $V_{U, p}$. This voltage is then reduced by $V_{s, p}$, due to the solid-phase ohmic resistance, and $V_{\eta, p}$, the surface overpotential. The electrolyte ohmic resistance then causes another drop $V_{e}$. At the negative electrode, there is a drop $V_{\eta, n}$ due to the surface overpotential, and a drop $V_{s, n}$ due to the solid-phase resistance. The voltage drops again due to the equilibrium potential at the negative current collector $V_{U, n}$. These voltages are described by the following set of equations:

$$
\begin{aligned}
V_{U, i} & =U_{0}+\frac{R T}{n F} \ln \left(\frac{1-x_{s, i}}{x_{s, i}}\right)+V_{\mathrm{INT}, i}, \\
V_{\mathrm{INT}, i} & =\frac{1}{n F}\left(\sum_{k=0}^{N_{i}} A_{i, k}\left(\left(2 x_{i}-1\right)^{k+1}-\frac{2 x_{i} k\left(1-x_{i}\right)}{\left(2 x_{i}-1\right)^{1-k}}\right)\right), \\
V_{o} & =i_{a p p} R_{o} \\
V_{\eta, i} & =\frac{R T}{F \alpha} \operatorname{arcsinh}\left(\frac{J_{i}}{2 J_{i 0}}\right) \\
J_{i} & =\frac{i}{S_{i}} \\
J_{i 0} & =k_{i}\left(1-x_{s, i}\right)^{\alpha}\left(x_{s, i}\right)^{1-\alpha} \\
V & =V_{U, p}-V_{U, n}-V_{o}^{\prime}-V_{\eta, p}^{\prime}-V_{\eta, n}^{\prime} \\
\dot{V}_{o}^{\prime} & =\left(V_{o}-V_{o}^{\prime}\right) / \tau_{o} \\
\dot{V}_{\eta, p}^{\prime} & =\left(V_{\eta, p}-V_{\eta, p}^{\prime}\right) / \tau_{\eta, p} \\
\dot{V}_{\eta, n}^{\prime} & =\left(V_{\eta, n}-V_{\eta, n}^{\prime}\right) / \tau_{\eta, n} .
\end{aligned}
$$

Here, $U_{0}$ is a reference potential, $R$ is the universal gas constant, $T$ is the electrode temperature (in $\mathrm{K}$ ), $n$ is the number of electrons transferred in the reaction ( $n=1$ for Li-ion), $F$ is Faraday's constant, $J_{i}$ is the current density, $J_{i 0}$ is the exchange current density, and $k_{i}$ is a lumped parameter of several constants including a 


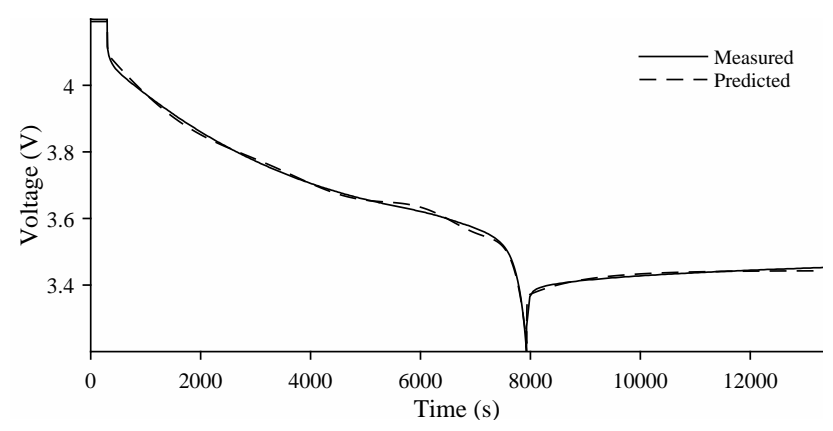

Figure 2. Battery voltage during 1 A discharge cycle.

rate coefficient, electrolyte concentration, and maximum ion concentration. $V_{\mathrm{INT}, i}$ is the activity correction term ( 0 in the ideal condition). We use the Redlich-Kister expansion with $N_{p}=12$ and $N_{n}=0$ (see ${ }^{1}$ ). The $\tau$ parameters are empirical time constants (used since the voltages do not change instantaneously).

The state vector, input vector, and output vector of the EOD model are defined follows:

$$
\begin{aligned}
\mathbf{x}_{\mathrm{EOD}}(t) & =\left[\begin{array}{lllllll}
q_{s, p} & q_{b, p} & q_{b, n} & q_{s, n} & V_{o}^{\prime} & V_{\eta, p}^{\prime} & V_{\eta, n}^{\prime}
\end{array}\right]^{T}, \\
\mathbf{u}(t) & =\left[i_{a p p}\right], \\
\mathbf{y}(t) & =[V] .
\end{aligned}
$$

Parameter values for a typical Li-ion cell are given in Daigle et al. ${ }^{1}$

An example discharge cycle is shown in Fig. 2. The cell starts fully charged at $4.2 \mathrm{~V}$ and is dishcarged at $1 \mathrm{~A}$. Around $8000 \mathrm{~s}$, the voltage hits the lower voltage limit, $3.2 \mathrm{~V}$, which defines EOD. At this point the load is removed and the cell voltage recovers back to the equilibrium potential. Here, we use $3.2 \mathrm{~V}$ as the voltage threshold defining EOD. In this case, the capacity is computed as the discharge time (7630 s) times the applied current $(1 \mathrm{~A})$, yielding in this case $2.12 \mathrm{Ah}$.

Note that capacity can only be measured consistently for a discharge cycle at reference conditions, since measured capacity is a nonlinear function of the load and environmental conditions. In this work, reference conditions are defined by a $1 \mathrm{~A}$ discharge at room temperature.

\section{Battery Aging Modeling}

Generally speaking, battery aging results in a loss of capacity and an increase in internal resistance. ${ }^{7}$ This is due to several physical processes such as solid electrolyte interface layer growth, which contributes to the resistance increase, diffusion stress, which leads to changes in diffusion properties and contributes to the capacity loss, and irreversible parasitic side reactions, which result in a loss of active Li-ions and contribute to the capacity loss. These aging phemonena have mainly been studied under constant loading conditions, but here, we want to estimate aging parameters based on real-life usage.

Battery behavior can also change over time due to faults, but here, we limit to normal degradation and aging processes. The effect of aging on a normal discharge cycle is shown in Fig. 3. Clearly, capacity decreases, since the time of EOD gets pushed earlier and earlier. Further, resistance is increased, accounting for the drop in voltage during discharge and also to the earlier EOD time. The diffusion parameter is also increasing, resulting in a slower diffusion rate, and thus the apparent state-of-charge (that observed at the electrode surface) decreases faster than with a normal diffusion rate, also contributing to the decrease in EOD time. Equilibrium potential after discharge is higher because less charge is drawn from the battery, due to the earlier EOD time and the decrease in diffusion rate (causing the $3.2 \mathrm{~V}$ limit to be reached sooner).

For the EOL model, we focus on how aging parameters change in time as a function of usage. Following our previous work, ${ }^{5}$ we select as the aging parameters $q^{\max }$, capturing the decrease in active Li-ions, and $R_{o}$, capturing the resistance increase, but, unlike our previous work, also consider the diffusion constant $D$, to capture changes in diffusion properties that can affect capacity. In the context of the EOD model, these 


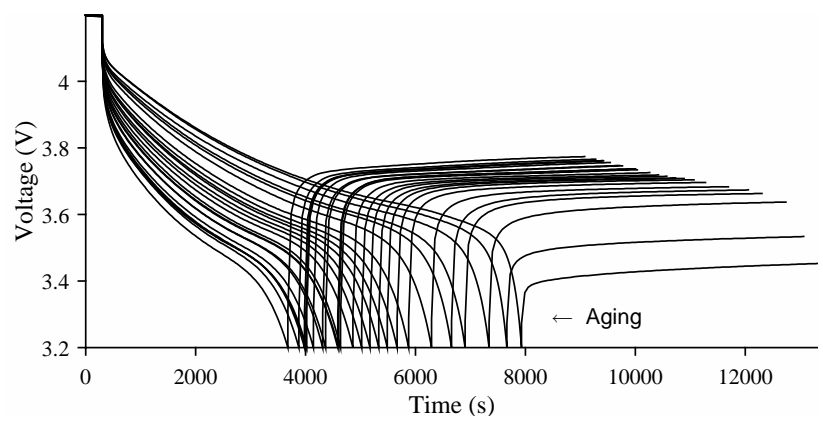

Figure 3. Battery voltage during 1 A discharge cycle as aging progresses.

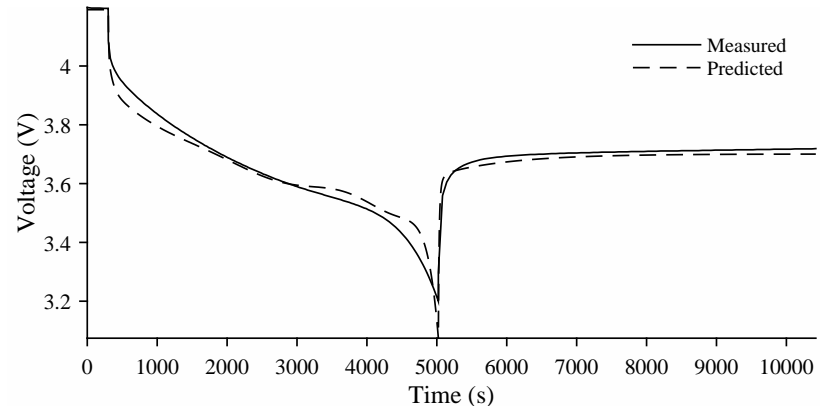

Figure 4. Battery voltage during 1 A discharge cycle halfway to EOL.

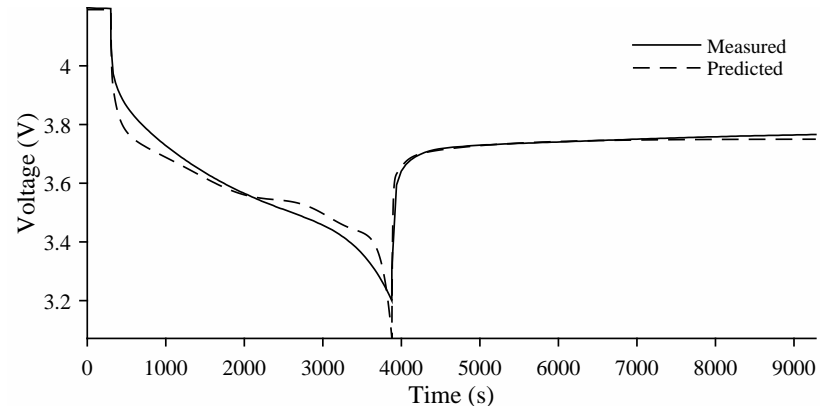

Figure 5. Battery voltage during 1 A discharge cycle at EOL.

parameters are constant, but in the context of the EOL model they are states:

$$
\mathbf{x}_{\mathrm{EOL}}(t)=\left[\begin{array}{lll}
q^{\max } & R_{o} & D
\end{array}\right]^{T} .
$$

Figs. 4 and 5 show how well changes to these parameters can capture the aging process, shown here for halfway to EOL and at EOL (where EOD, and thus measured capacity, is half of nominal). Although there are some differences, the time of EOD and the equilibrium potential after discharge are captured very accurately.

These variables change as a function of battery usage, i.e., as a function of the applied current, $i_{a p p} \cdot{ }^{7}, 17$ We assume that the following models descibing their dynamics:

$$
\begin{aligned}
\dot{q}^{\max } & =w_{q}\left|i_{a p p}\right|, \\
\dot{R}_{o} & =w_{R}\left|i_{\text {app }}\right|, \\
\dot{D} & =w_{D}\left|i_{\text {app }}\right|,
\end{aligned}
$$

where the $w$ parameters are the aging rate parameters, which are constants. If $w_{q}, w_{R}, w_{D}$, and future values of $i_{a p p}$ are known, then we can predict future values of $\mathbf{x}_{\mathrm{EOL}}$, and given that, determine whether the

$$
6 \text { of } 11
$$



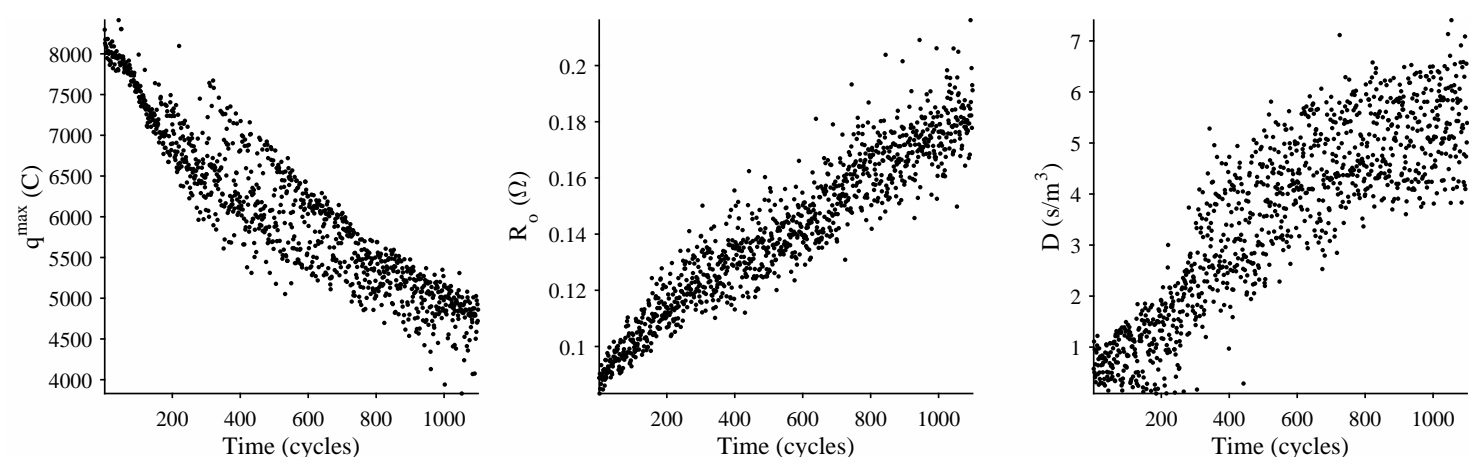

Figure 6. Estimates of aging parameters over discharge cycles.

capacity as measured at reference conditions would be below the limit. Here, we use a $50 \%$ of the nominal capacity as the threshold defining EOL.

Our experiments consist of a sequence of charge and discharge cycles at randomized load. Since capacity can only be measured for a discharge at reference conditions, reference discharge cycles have been performed at regular intervals in order to obtain a ground truth EOL. Note that in practical usage scenarios, reference discharges will not be available and capacity can only be estimated from the estimates of the aging parameters, $\mathbf{x}_{\mathrm{EOL}}$.

\section{Estimation}

In this section we describe the estimation procedures. As shown in Fig. 1, there are three estimation problems: (i) state estimation for the EOD model, (ii) state estimate for the EOL model, and (iii) parameter estimation for the EOL model. We describe each of these in turn.

\section{A. State Estimation for the EOD Model}

State estimation for the EOD model has been developed in previous work, ${ }^{1,11}$ so here we only summarize the approach. Here, we have a standard state estimation problem, with the model described by that in Section III and the states given by $\mathbf{x}_{\mathrm{EOD}}$. Since the model is nonlinear, a nonlinear filter is needed. For this purpose, we use the unscented Kalman filter (UKF) ${ }^{18,19}$ due to its high accuracy and low computational cost. The UKF uses the concept of sigma points, which are deterministically selected samples that are selected to represent the first two statistical moments of a distribution. The unscented transform is the procedure that selects sigma points from a given distribution. By passing sigma points through a nonlinear function, an approximation to the first two statistical moments of the original distribution passed through that function is computed. The filter first updates the current state estimate using the state equation, then corrects the estimate based on the observations, through the use of the sigma points. Details of the algorithm and its application to prognostics are provided elsewhere. ${ }^{18-20}$

\section{B. State Estimation for the EOL Model}

For the EOL model, the state estimation problem is to estimate $\mathbf{x}_{\mathrm{EOL}}$, i.e., $q^{\max }, R_{o}$, and $D$. In this case, we assume these parameters are approximately constant over a given discharge cycle, and so use system identification techniques to estimate their values for a given discharge cycle. For this, we use the NedlerMead algorithm for multi-dimensional unconstrained nonlinear minimization. For given values of the aging parameters, the model is simulated and the sum of the mean-squared error is computed between the predicted voltage and the observed voltage. The optimization algorithm searches over values of the aging parameters to minimize this error. The algorithm is very fast, and finds a solution in under $20 \mathrm{~s}$ on average. For optimal results, the discharge cycle data must include the rest period after reaching EOD, in order to correctly characterize $q^{\max }$.

This procedure is repeated after every discharge cycle. As the battery ages, the estimated values of these parameters change in time. Fig. 6 shows the results of this procedure on a battery undergoing random walk 


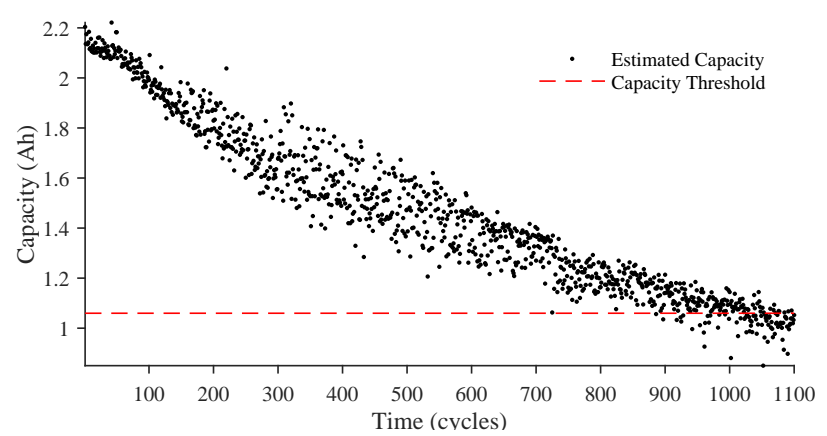

Figure 7. Estimated capacity as a function of estimated aging parameters.
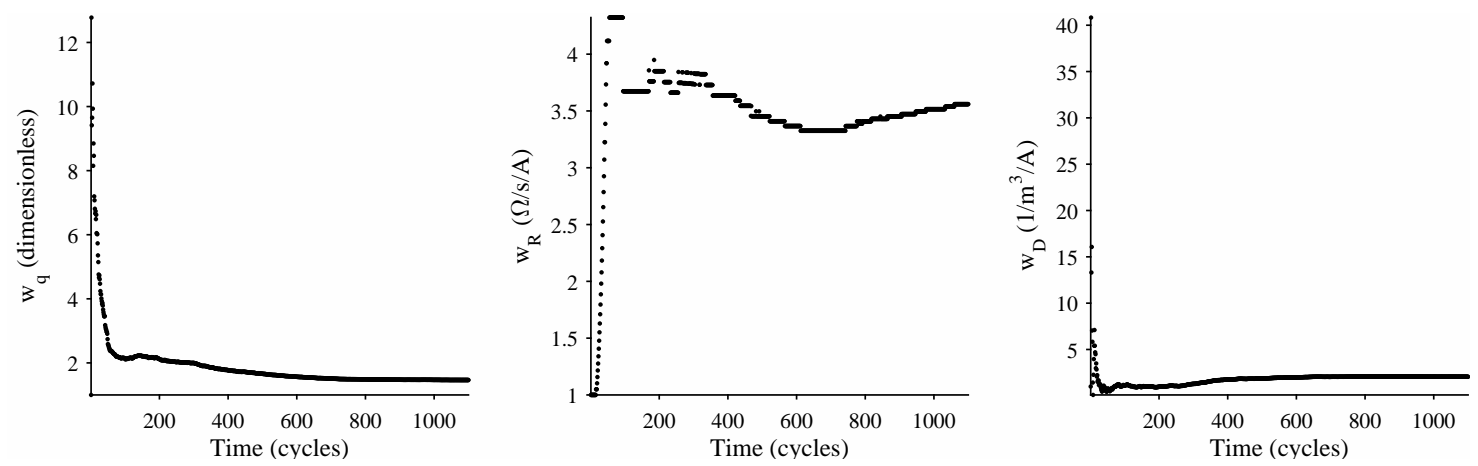

Figure 8. Estimates of aging rate parameters over discharge cycles.

discharge cycles. The estimates are fairly noisy, however a clear trend is observable: $q^{\text {max }}$ decreases with age, $R_{o}$ increases with age, and $D$ increases with age.

For given values of the aging parameters, we can simulate a discharge cycle at reference conditions to compute a corresponding value of estimated capacity. Fig. 7 shows the estimated capacity as a function of the estimated aging parameter values after each discharge cycle. Capacity is most sensitive to changes in $q^{\max }$.

\section{Parameter Estimation for the EOL Model}

For estimation of the aging rate parameters $w_{q}, w_{R}$, and $w_{D}$, we try to fit values of these parameters to the estimated values of the aging parameters. In a discrete-time form, the equation describing $q^{\max }(k)$ is

$$
q^{\max }(k+1)=q^{\max }(k)+w_{q}(k)\left|i_{a p p}(k)\right| d t(k) .
$$

Note that the discrete time points are after every discharge cycle, and, hence, are not evenly spaced, i.e., the time interval $d t(k)$ is not constant. Between discharge cycles, the applied current is variable, so for $\left|i_{a p p}(k)\right|$ we take an average of the current for the $k$ th discharge cycle (and the preceding charge cycle). The formulation is equivalent for $R_{o}(k)$ and $D(k)$.

Again, we use the Nedler-Mead algorithm, using the estimated values of the aging parameters from the initial value of $k, k_{0}$, up to the current value. After each new estimate of the aging parameters, we update the aging rate parameter estimates based on all available aging parameter estimates up to the current discharge cycle. With more data, the estimate becomes more accurate and converges. The estimates of the aging rate parameters are shown in Fig 8. The parameter estimates converge after about 100-200 cycles.

Fig. 9 shows the predicted values of the aging parameters based on the aging models using final estimated values of the aging rate parameters, against their estimated values. These aging models describe the evolution of the aging parameters as the battery ages fairly well. 

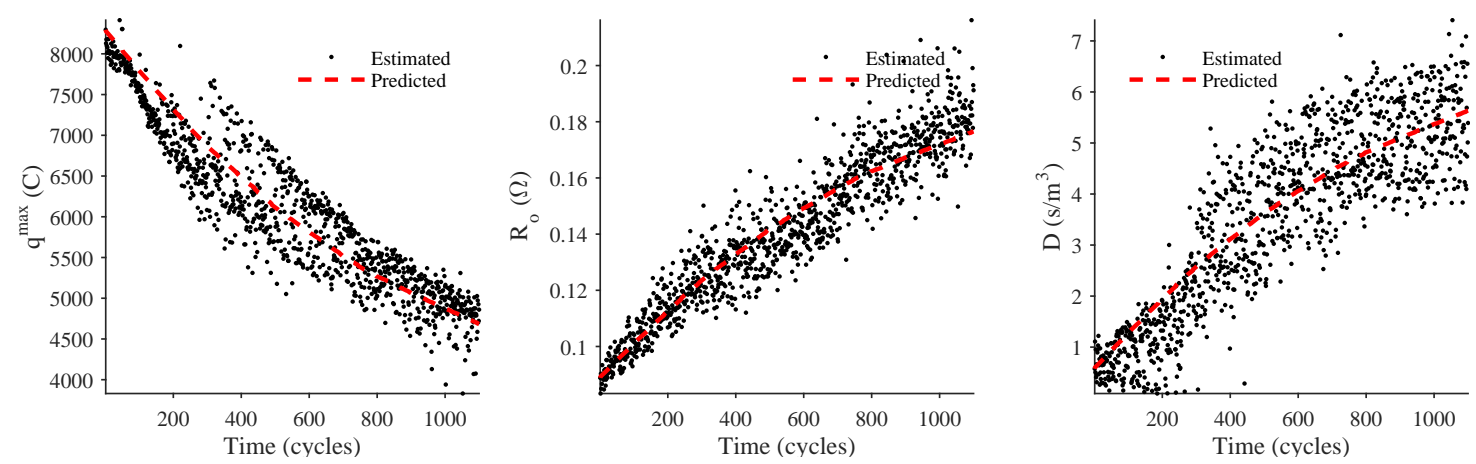

Figure 9. Estimates and predictions of aging parameters over discharge cycles.
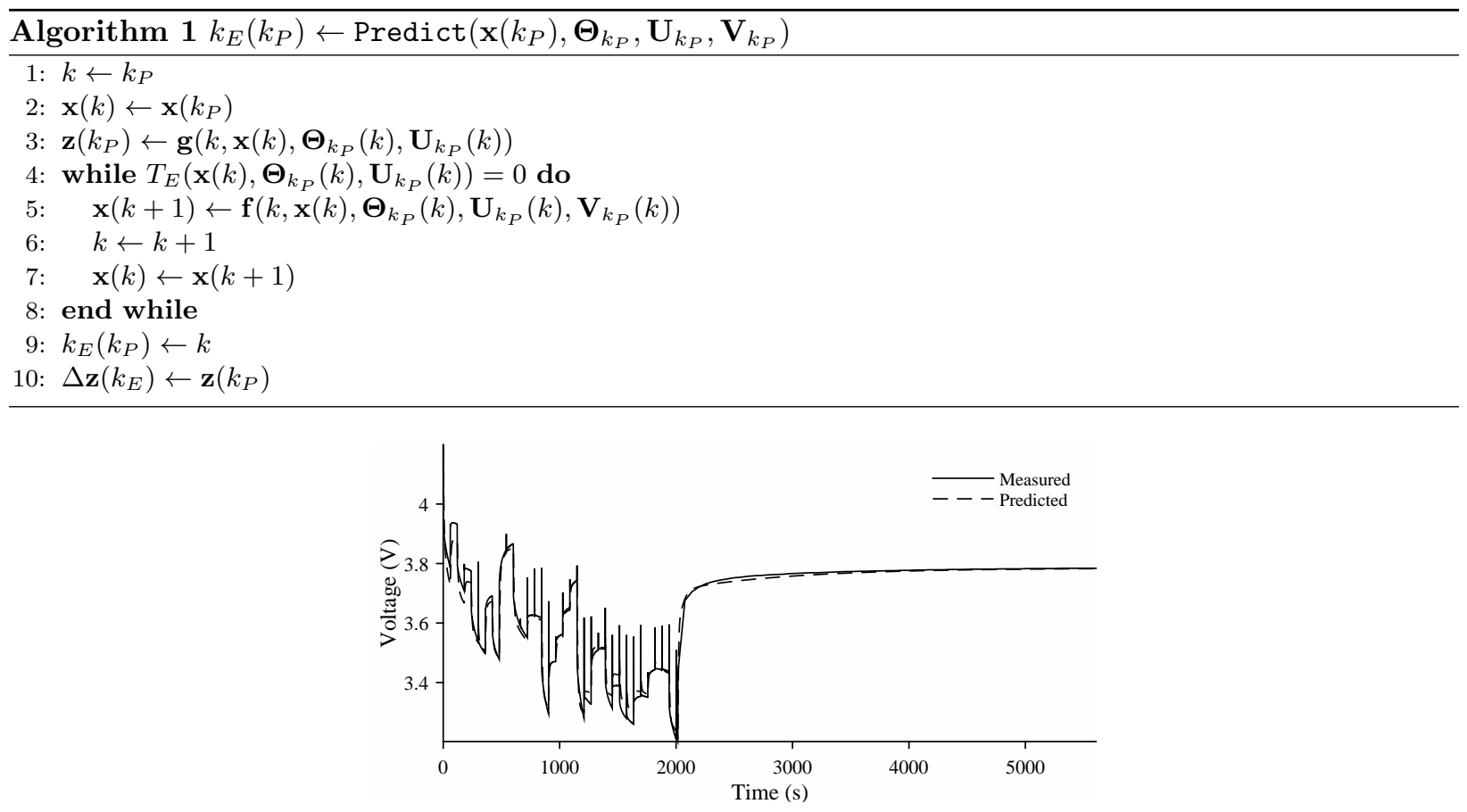

Figure 10. Battery voltage during random walk discharge cycle halfway to EOL.

\section{Prediction}

There are two different prediction problems to consider, EOD prediction and EOL prediction. We use the same algorithm in both cases, which is basically to simulate the given model forward for given intial state and parameter values, and hypothesized future inputs and process noise. The core procedure is shown as Algorithm 1. If probability distributions are given, then this algorithm can be used on realizations of the prediction inputs within an algorithm such as Monte Carlo sampling.

For both EOD and EOL prediction, we assume that the future usage is known, and process noise is negligible. The case where future usage is described by a probability distribution is covered in other works. ${ }^{11,13}$ For EOD prediction, we assume there are no unknown parameters (the values used for the aging parameters are the most recently estimated by the EOL model state estimation algorithm), and for EOL prediction, we assume the aging rate parameters are constant and their values given by the most recently estimated values by the EOL parameter estimation algorithm.

As seen in Figs. 2-5, when the current to be applied is known, accurate predictions of EOD can be computed even at $t=0$. An open loop prediction for a random walk discharge is shown in Fig. 10, and is also very accurate in predicting EOD in the open loop; EOD is predicted to be only 5 s later than it actually 


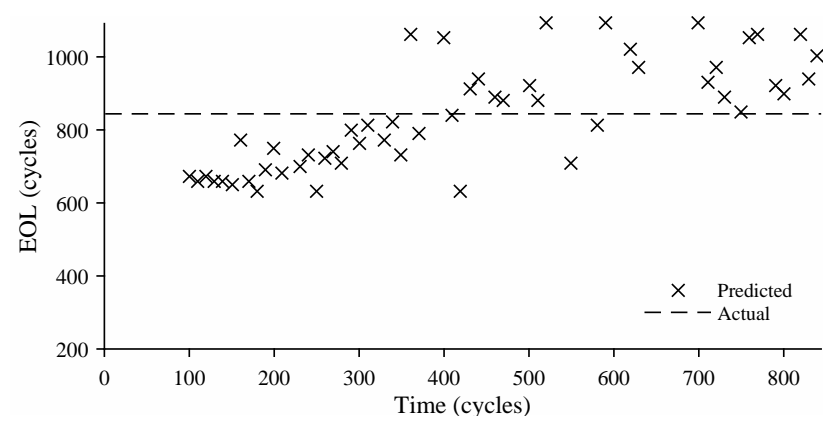

Figure 11. EOL predictions.

occurs. Predictions at later times are only more accurate. However, as the battery ages, there is a bias that appears in the EOD predictions, and EOD is overestimated. As a result, capacity estimates will be biased and capacity will be overestimated. This means that additional parameters need to be considered to change as a function of aging, in order to more accurately characterize the EOD time at higher battery ages, since the three selected aging parameters are not sufficient to eliminate that bias.

For EOL prediction, at any prediction point, we have the current estimated values of the aging parameters and aging rate parameters. We can use these and the EOL model to predict the future values of the aging parameters and to see when these future values of the aging parameters will result in the capacity being less than the limit. Results are shown in Fig. 11. The true EOL is determined to be at the 844th discharge cycle. At each prediction point, we use as the initial state the estimated aging parameters at that cycle and use the estimated aging rate parameters computed at that cycle. Since the EOD prediction is biased at higher ages, the capacity estimates are also biased and the EOL predictions are biased. With a better parameter fit at higher ages, this bias can be eliminated.

\section{Conclusions}

In this paper we proposed a new framework for EOD and EOL prediction for Li-ion batteries, proposing new aging models. Procedures for estimation and prediction are described. The overall approach can estimate the current battery state, perform EOD prediction using up-to-date aging parameters, and perform EOL prediction using aging models and estimated aging rate parameters.

Here, we selected a critical subset of battery parameters and observed how they change in age through system identification procedures applied over any given discharge cycle. Based on the EOL prediction results, we found that additional parameters must be considered as aging parameters in order to better characterize EOD at higher ages. This would result in better (unbiased) capacity estimates and correspondingly better EOL predictions. In addition, no filtering was performed on the aging parameter estimates, but this would result in more precise capacity estimates and EOL predictions. Uncertainty in the states, parameters, and future usage was also not considered here, and is also a subject of future work.

\section{Acknowledgements}

This work was supported by the SMART-NAS project under the Airspace Operations and Safety (AOSP) program within the Aeronautics Research Mission Directorate (ARMD). The authors gratefully acknowledge Brian Bole for obtaining the experimental data used in this work.

\section{References}

\footnotetext{
${ }^{1}$ Daigle, M. and Kulkarni, C., "Electrochemistry-based Battery Modeling for Prognostics," Annual Conference of the Prognostics and Health Management Society 2013, Oct. 2013, pp. 249-261.

${ }^{2}$ Saha, B., Goebel, K., Poll, S., and Christophersen, J., "Prognostics methods for battery health monitoring using a Bayesianframework," IEEE Transactions on Instrumentation and Measurement, Vol. 58, No. 2, Feb. 2009, pp. $291-296$.

${ }^{3}$ Sweet, A., Gorospe, G., Daigle, M., Celaya, J., Balaban, E., Roychoudhury, I., and Narasimhan, S., "Demonstration of Prognostics-Enabled Decision Making Algorithms on a Hardware Mobile Robot Test Platform," Annual Conference of the
} 
Prognostics and Health Management Society 2014, September 2014, pp. 142-150.

${ }^{4}$ Saha, B. and Goebel, K., "Modeling Li-ion battery capacity depletion in a particle filtering framework," Proceedings of the Annual Conference of the Prognostics and Health Management Society 2009, Sept. 2009.

${ }^{5}$ Bole, B., Kulkarni, C., and Daigle, M., "Adaptation of an Electrochemistry-based Li-Ion Battery Model to Account for Deterioration Observed Under Randomized Use," Annual Conference of the Prognostics and Health Management Society 2014, September 2014, pp. 502-510.

${ }^{6}$ Ning, G. and Popov, B. N., "Cycle life modeling of lithium-ion batteries," Journal of The Electrochemical Society, Vol. 151, No. 10, 2004, pp. A1584-A1591.

${ }^{7}$ Ning, G., White, R. E., and Popov, B. N., "A generalized cycle life model of rechargeable Li-ion batteries," Electrochimica Acta, Vol. 51, No. 10, 2006, pp. 2012-2022.

${ }^{8}$ Peterson, S. B., Apt, J., and Whitacre, J. F., "Lithium-ion battery cell degradation resulting from realistic vehicle and vehicle-to-grid utilization," Journal of Power Sources, Vol. 195, No. 8, 2010, pp. 2385-2392.

${ }^{9}$ Bole, B., Daigle, M., and Gorospe, G., "Online Prediction of Battery Discharge and Estimation of Parasitic Loads for an Electric Aircraft," Second European Conference of the Prognostics and Health Management Society 2014, July 2014, pp. $23-32$.

${ }^{10}$ Quach, C., Bole, B., Hogge, E., Vazquez, S., Daigle, M., Celaya, J., Weber, A., and Goebel, K., "Battery Charge Depletion Prediction on an Electric Aircraft," Annual Conference of the Prognostics and Health Management Society 2013, October 2013, pp. 503-512.

${ }^{11}$ Daigle, M., Sankararaman, S., and Kulkarni, C., "Stochastic Prediction of Remaining Driving Time and Distance for a Planetary Rover," 2015 IEEE Aerospace Conference, March 2015.

${ }^{12}$ Daigle, M. and Goebel, K., "Model-based Prognostics with Concurrent Damage Progression Processes," IEEE Transactions on Systems, Man, and Cybernetics: Systems, Vol. 43, No. 4, May 2013, pp. 535-546.

${ }^{13}$ Daigle, M. and Sankararaman, S., "Advanced Methods for Determining Prediction Uncertainty in Model-Based Prognostics with Application to Planetary Rovers," Annual Conference of the Prognostics and Health Management Society 2013, October 2013, pp. 262-274.

${ }^{14}$ Sankararaman, S., Daigle, M., and Goebel, K., "Uncertainty Quantification in Remaining Useful Life Prediction using First-Order Reliability Methods," IEEE Transactions on Reliability, Vol. 63, No. 2, June 2014, pp. 603-619.

${ }^{15}$ Rahn, C. D. and Wang, C.-Y., Battery Systems Engineering, Wiley, 2013.

${ }^{16}$ Karthikeyan, D. K., Sikha, G., and White, R. E., "Thermodynamic model development for lithium intercalation electrodes," Journal of Power Sources, Vol. 185, No. 2, 2008, pp. 1398-1407.

${ }^{17}$ Park, M., Zhang, X., Chung, M., Less, G. B., and Sastry, A. M., "A review of conduction phenomena in Li-ion batteries," Journal of Power Sources, Vol. 195, No. 24, 2010, pp. 7904-7929.

${ }^{18}$ Julier, S. J. and Uhlmann, J. K., "A new extension of the Kalman filter to nonlinear systems," Proceedings of the 11th International Symposium on Aerospace/Defense Sensing, Simulation and Controls, 1997, pp. 182-193.

${ }^{19}$ Julier, S. J. and Uhlmann, J. K., "Unscented filtering and nonlinear estimation," Proceedings of the IEEE, Vol. 92, No. 3, Mar 2004, pp. 401-422.

${ }^{20}$ Daigle, M., Saha, B., and Goebel, K., "A Comparison of Filter-based Approaches for Model-based Prognostics," 2012 IEEE Aerospace Conference, March 2012. 